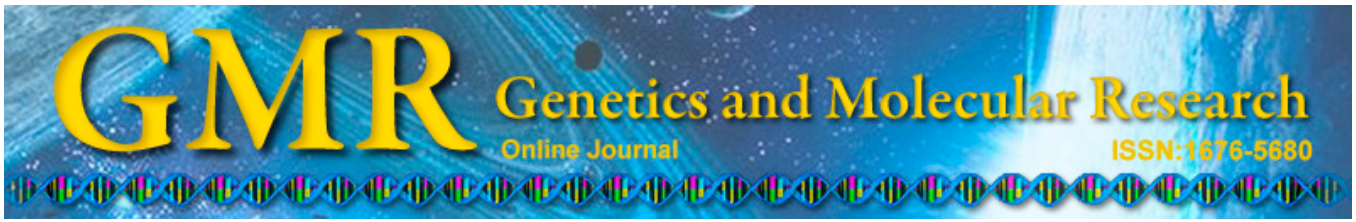

\title{
Curative effect and costs of surgical and gamma knife treatments on intractable epilepsy caused by temporal-hippocampal sclerosis
}

\author{
Z.T. Han and Q.X. Chen \\ Neurosurgery Department, Renmin Hospital of Wuhan University, \\ Hubei Wuhan Zhangzhidong, Hubei, China \\ Corresponding author: Q.X. Chen \\ E-mail: chenqianxuerhwu@163.com
}

Genet. Mol. Res. 14 (3): 8555-8562 (2015)

Received November 3, 2014

Accepted May 14, 2015

Published July 31, 2015

DOI http://dx.doi.org/10.4238/2015.July.31.3

\begin{abstract}
This study aimed to investigate the curative effect and costs of surgical and gamma knife treatments on intractable epilepsy caused by temporal-hippocampal sclerosis. The subjects comprised patients who suffered from intractable epilepsy caused by temporalhippocampal sclerosis and received treatment in the Department of Neurosurgery of our hospital between 2010 and 2011. After obtaining their consent, patients were evaluated and selected to receive surgical or gamma knife treatments. In the surgical group, the short-term curative rate was $92.60 \%$ and the average cost was US\$ 1311.50 while in the gamma knife group, the short-term curative rate was $53.79 \%$, and the average cost was US\$ 2786.90 . Both surgical and gamma knife treatments of intractable epilepsy caused by temporal-hippocampal sclerosis are safe and effective,
\end{abstract}


but the short-term curative effect of surgical treatment is better than that of gamma knife, and its cost is lower.

Key words: Temporal-hippocampal sclerosis; Intractable epilepsy; Surgery; Gamma knife

\section{INTRODUCTION}

Hippocampal sclerosis is a pathological change mainly manifested as hippocampal neuron loss and mossy fiber sprouting. Studies have found that the loss of hippocampal neurons leads to synaptic reorganization of the surviving cells, so that the synchronization between the cells and their excitability increases abnormally, resulting in spontaneous seizures (Ozkara and Aronica, 2012). The reasons for the loss of hippocampal neurons have been discussed controversially over the years. Recent studies suggest that early risk factors in childhood, susceptibility to genetic epilepsy and dysplasia are the main reasons (Svenstrup et al., 2011). The incidence of hippocampal sclerosis (HS) peaks at about 10 years of age. Once the disease enters the attack stage of intractable epilepsy, seizures gradually become difficult to control after several months to a few years of treatment. This causes neuropsychiatric symptoms such as personality disorders and cognitive function retrogression, and a decline in learning and memory function (Malmgren and Thom, 2012). Treatment with anti-epileptic drugs is effective for the majority of patients in an early stage, but the disease develops to intractable epilepsy gradually around puberty or earlier, when the combination of multiple drugs is often unable to control the seizures and the long-term prognosis is relatively poor. With the long-term recurrent episodes, patients gradually experience interictal behavior disorders, learning disabilities and social problems. For the patients in whom seizures are difficult to control by regular anti-epileptic drugs, surgery should be considered as soon as possible (Porter, 2012). Medial temporal lobectomies, which can eliminate or significantly reduce seizures and improve the quality of life of the patients, are effective for $90 \%$ of patients with medial temporal lobe epilepsy (MTLE). Although surgery can eliminate seizures in the late stage, it is difficult to reverse mental and behavior disorders (Usami et al., 2012).

At present, there is a consensus to adopt surgical treatment in intractable epilepsy caused by HS. There are a number of reports on the preoperative evaluation, safety and effectiveness of surgery (Ferrari-Marinho et al., 2012). Recently all major hospitals in the United States have been exploring the use of gamma knife in the treatment of HS (Prayson and Yoder, 2007). In the present study, 44 patients who suffered from intractable epilepsy caused by temporal-hippocampal sclerosis and received treatment in the Department of Neurosurgery of our hospital between 2010 and 2011 were selected after evaluation. Among these, 30 patients received surgical lesion resection and 14 received gamma knife lesion irradiation. We conducted follow-ups and summarized the effects of the two treatments as follows.

\section{MATERIAL AND METHODS}

\section{Subjects}

Forty-five patients suffering from medial temporal lobe epilepsy were selected. These 
patients' epilepsy could not be controlled after receiving formal anti-epileptic treatment in the Department of Neurosurgery of our hospital between 2010 and 2011.

\section{Preoperative assessment items}

Magnetic resonance imaging (MRI) and video-electroencephalography monitoring (V/EEG) were performed during seizures and the interictal period, and single-photon emission computed tomography scans were also performed in some patients. After the assessment, the subjects were diagnosed with intractable epilepsy caused by hippocampal sclerosis. Among the 44 patients, 14 received gamma knife treatment, and 30 received craniotomy. Wada tests were conducted on the patients for craniotomy treatment who had a partial right lesion and complete left lesion, and the patients who passed the test underwent surgery. EEG 7 review was performed on the patients in both groups 7 days after treatment, and EEG or MRI reviews were conducted later depending on their disease changes. EEG reviews were performed 3 and 6 months after the surgery.

\section{Evaluation criteria}

According to Thom et al., (2010): 1) No disease recursion in the recent 6 months (the cases with symptomatic signs were excluded);2) The frequency of disease onset was lowered by at least $50 \%$ compared to the preoperative 6 months; 3 ) The frequency of disease onset was lowered by less than $50 \%$ compared to the preoperative 6 months; 4) Disease onset was not significantly lowered; 5) Disease onset was accelerated. The result of itens 1 and 2 was considered effective.

\section{Grouping and case description}

Grouping and case descriptions are shown in Table 1.

Table 1. Grouping and case description.

\begin{tabular}{lccccc}
\hline & Male & Female & Age of disease onset (years) & Surgical age (years ) & Average follow-up time \\
\hline Surgical group $(\mathrm{N}=30)$ & 19 & 11 & $10.39 \pm 5.10$ & $25.65 \pm 10.25$ & 6-32 months (median: 8 months) \\
Gamma knife group $(\mathrm{N}=14)$ & 8 & 6 & $10.81 \pm 8.10$ & $23.34 \pm 6.05$ & $12-53$ months (median: 31 months) \\
\hline
\end{tabular}

\section{RESULTS}

\section{History and type of seizure}

The history and types of seizure are summarized in Table 2 . 
Z.T. Han and Q.X. Chen

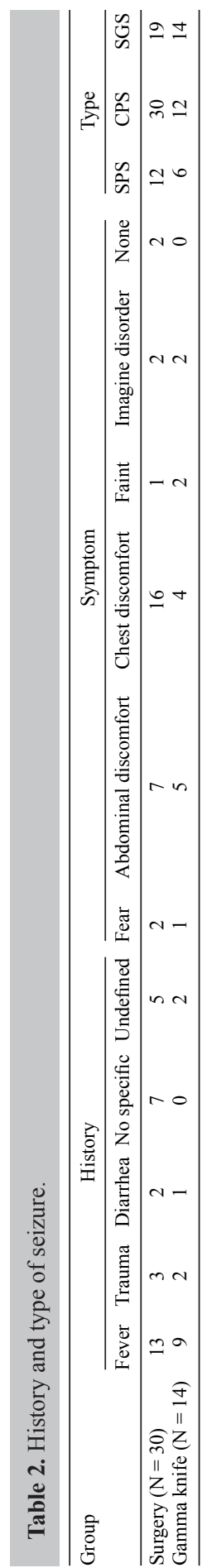




\section{Results of auxiliary examinations}

1) The 44 patients were subjected to transcranial MRI examination, and recorded interictal EEG, including sphenoid electrode tracings.

2) For the patients with interictally bilateral migratory epileptiform discharges contrary to MRI lesions, we performed the V/EEG during seizures.

3) There were 16 patients with left HS in the surgery group. With the exception of the first patient for the surgical treatment showing hippocampal gliosis by MRI and two cases of left-handed patients, the remaining 14 patients were subjected to Wada tests before the surgery after carotid injection with clonazepam of 2 to $3 \mathrm{mg}$ (Tezer et al., 2011).

\section{Results of MRI and interictal EEG and V/EEG}

1) Among the 44 patients, 18 patients suffering from left HS and 26 patients, right HS.

2) Interictal EEGs, including sphenoid electrode tracings were carried out in all the patients. There were 25 cases in which interictal epileptiform discharges were always at the same side, 17 cases of bilateral migration, and only 2 cases of contra-lateral MRI foci.

3) For the patients whose interictally bilateral migratory epileptiform discharges were contrary to MRI lesions, V/EEG was performed during seizures, confirming that the initiation site of interictal epileptiform discharges were on the same side as the MRI lesions. For the patients whose ipsilateral epileptiform discharges were inconsistent with the structural changes of MRI, we made a prudent choice to continue medication.

4) In the surgical treatment group, there were 12 patients that did not have any seizures during EEG recordings, in which 7 patients were subject to long-term EEG monitoring for 12 to $42 \mathrm{~h}$ and at least two records of complete sleep process at night. Overall, the ratio of epileptiform discharges on the MRI lesion side to those on its contra-lateral side was larger than $93 \%$. Three patients underwent interictal EEG recordings 2 to 14 times. Their clinical manifestations and MRI were typical CPS (complex partial seizure) caused by HS, so they received surgical treatment directly and all of them gained a short-term post-operative effect completely without seizures (Marucci, 2011).

\section{Wada test results}

There were 16 patients with left HS in the surgery group. Excluding a patient who exhibited gliosis during the MRI and two cases of left-handed patients, the remaining 14 patients were subject to Wada tests preoperatively, using carotid injections of clonazepam at a dose of 2 to $3 \mathrm{mg}$. Twelve patients passed the test at one time $(\geq 5 / 6)$, and 3 cases exhibited results of less than or equal to 4/6 in bilateral lesions, identifying that the patients had temporal lobe lesions involved in memory and language, and then they received the surgery (Kagawa et al., 2012).

\section{Follow-up results}

The follow-up results are shown in Table 3. 


\begin{tabular}{l} 
Table 3. Follow-up results $\left(\times 10^{-2}\right)$. \\
\hline Group \\
\cline { 2 - 8 }
\end{tabular}

\section{Efficacy comparison}

1) Both surgical lesion excision and gamma knife lesion irradiation were safe and effective treatment methods for medically intractable epilepsy caused by hippocampal sclerosis. However, the short-term effect of surgical treatment (92.60\%) was better than that of gamma knife treatment $(53.79 \%)(\mathrm{P}=0.0014)$. In addition, the effect/cost performance of the former was higher than that of the latter.

2) The effect of surgical therapy appeared immediately after the surgery, while that of Gamma knife treatment usually showed a few years or several months later.

3) There was no occurrence of delayed cerebral edema after the surgery. In contrast, the patients under gamma knife treatment sometimes undergo recurrent disease several months after the treatment, often accompanied by cerebral edema, which was required to use drug therapy, including corticosteroid and dehydrating agent. There was no obvious relationship between the occurrence of cerebral edema and radiation dose for treatment.

\section{DISCUSSION}

In this study, the follow-up time of the patients was statistically different between the two groups, with a median 33 months in the gamma knife group and 10 months in the surgical treatment group. The effect of gamma knife treatment reported in the literature rarely appears immediately after treatment, but usually 8 to 15 months later (Srikijvilaikul et al., 2004). According to the latest foreign statistical data on long-term follow-up cases, although the effect of anteromedial temporal resection drifts over time, the overall trend is stable and durable (Hoshida, 2011).

Medically intractable epilepsy caused by HS should be treated surgically as soon as possible, which has become an indisputable principle in the field of medicine. Its safety and efficacy is equivalent to that of surgical appendectomy (Lerche et al., 2011).

Patients with refractory complex partial seizures (with or without secondary comprehensive attack), in whom the treatment of the first-line anti-epileptic drug fails, should be introduced to the epilepsy surgical treatment center. If the center determines that the patients fulfill the standard for anteromedial temporal lobe resection, and the patients accept the consequences of surgery and possible risks, surgical treatment should be adopted, rather than continue treatment with drugs. As there has been no sufficient evidence to confirm or deny the efficacy of local neocortex resection in patients, recommendations for surgery are not possible (Patil and Andrews, 2010).

The gamma knife group showed a significantly lower treatment effect than that of the surgery group and had more frequent recurrent post-operative attacks in the long-term. The patients in the gamma knife group underwent post-operatively delayed cerebral edema, and the 
duration and time of occurrence had no obvious regularity, so the patient continued to suffer from pain and experience a lower quality of life. We suggest that the difference in the effects between surgery and gamma knife treatments for medial temporal lobe epilepsy be regarded correctly, so as to appropriately guide patients. The patients without surgical contraindications should be guided to receive surgical treatment as much as possible.

Some practices for reference in the actual work and problems needing attention are as follows: the majority of patients in our country are still in poverty. Therefore, under conditions of limited medical resources, we must treat patients who can afford hospital services. The priority would be to "find the correct diagnosis, offer the right guidance and achieve cost-effective performance" to solve their problems in a feasible and effective way. In order to achieve the above, the following aspects should be considered:

1) The first step involves improving the understanding and diagnosis of MTLE, through EEG and MRI clinically is the first step to ensure patients to get correct guidance: A. In this study, we were not aware that $92 \%$ of the patients had seizures caused by MTLE. Therefore, it is important to enhance the diagnosis of MTLE from symptomatology (Henry et al., 2011). B. Use of sphenoidal electrodes in the EEG examination could improve the diagnostic significance of EEG during the interictal period. C. The level of MRI examination and filmreading capability of surgeons should be improved. Early MRIs, including coronal scans of the temporal lobe, must be performed on patients with suspected MTLE to introduce a certain quantitative indication (Vale et al., 2012).

2) Patients should be provided with appropriate guidance, so that they and their families know about the safety and efficacy of anteromedial temporal lobe resection.

3) High effect/cost performance: The results of the study showed that the effect/cost performance of the surgery group was higher than that of the gamma knife treatment group. This preliminary data should be provided to patients before choosing treatment methods.

\section{REFERENCES}

Ferrari-Marinho T, Caboclo LO, Marinho MM, Centeno RS, et al. (2012). Auras in temporal lobe epilepsy with hippocampal sclerosis: relation to seizure focus laterality and post surgical outcome. Epilepsy Behav. 24: 120-125.

Henry TR, Chupin M, Lehéricy S, Strupp JP, et al. (2011). Hippocampal sclerosis in temporal lobe epilepsy: findings at 7 T(1). Radiology 261: 199-209.

Hoshida T (2011). Surgery for temporal lobe epilepsy: historical review and postoperative results. Brain Nerve 63: 313-320.

Kagawa K, Iida K, Katagiri M, Nishimoto T, et al. (2012). Successful treatment of mesial temporal lobe epilepsy with bilateral hippocampal atrophy and false temporal scalp ictal onset: a case report. Hiroshima J. Med. Sci. 61: 37-41.

Lerche H, Vezzani A, Beck H, Blumcke I, et al. (2011). New developments in epileptogenesis and therapeutic perspectives. Nervenarzt 82: 978-985.

Malmgren K and Thom M (2012). Hippocampal sclerosis-origins and imaging. Epilepsia 53 (Suppl 4): 19-33.

Marucci G (2011). Commentary on patterns of hippocampal sclerosis as predictors of postsurgical outcome. Epilepsia 52: $652-653$

Ozkara C and Aronica E (2012). Hippocampal sclerosis. Handb. Clin. Neurol. 108: 621-639.

Patil AA and Andrews RV (2010). Nonresective hippocampal surgery for epilepsy. World Neurosurg. 74: 645-649.

Porter RJ (2012). Addressing the need for new therapies for epilepsy. Epilepsia 53: 1840-1841.

Prayson RA and Yoder BJ (2007). Clinicopathologic findings in mesial temporal sclerosis treated with gamma knife radiotherapy. Ann. Diagn. Pathol. 11: 22-26.

Srikijvilaikul T, Najm I, Foldvary-Schaefer N, Lineweaver T, et al. (2004). Failure of gamma knife radiosurgery for mesial temporal lobe epilepsy: report of five cases. Neurosurgery 54: 1395-1402.

Svenstrup K, Møller RS, Christensen J, Budtz-Jørgensen E, et al. (2011). NIPA1 mutation in complex hereditary spastic paraplegia with epilepsy. Eur. J. Neurol. 18: 1197-1199.

Tezer FI, Dericioglu N, Bozkurt G, Bilginer B, et al. (2011). Epilepsy surgery in patients with unilateral mesial temporal 
sclerosis and contralateral scalp ictal onset. Turk. Neurosurg. 21: 549-554.

Thom M, Mathern GW, Cross JH and Bertram EH (2010). Mesial temporal lobe epilepsy: How do we improve surgical outcome? Ann. Neurol. 68: 424-434.

Usami K, Kawai K, Koga T, Shin M, et al. (2012). Delayed complication after Gamma Knife surgery for mesial temporal lobe epilepsy. J. Neurosurg. 116: 1221-1225.

Vale FL, Bozorg AM, Schoenberg MR, Wong K, et al. (2012). Long-term radiosurgery effects in the treatment of temporal lobe epilepsy. J. Neurosurg. 117: 962-969. 